\title{
Measurement of Hearing Impairment among Greenlandic School-Children: Association Between Self-Reported Data and Clinical Examinations
}

\section{Christina Schnohr ( $\sim$ cwsc@sund.ku.dk)}

Department of Public Health, University of Copenhagen https://orcid.org/0000-0002-3068-9879 Jakob Schmidt Jensen

Copenhagen University Hospital: Rigshospitalet

\section{Cecilie Friis Skovsen}

University of Copenhagen: Kobenhavns Universitet

\section{Preben Homøe}

Zealand University Hospital Koge: Sjaellands Universitetshospital Koge

\section{Birgit Niclasen}

\author{
Allorfik
}

\section{Ramon Gordon Jensen}

Copenhagen University Hospital: Rigshospitalet

\section{Research article}

Keywords: Hearing impairment, prevalence, measurement properties, methodology, school-children, Greenland.

Posted Date: June 30th, 2021

DOl: https://doi.org/10.21203/rs.3.rs-658963/v1

License: (9) This work is licensed under a Creative Commons Attribution 4.0 International License. Read Full License 


\section{Abstract}

Background: Ear infections is the leading cause of hearing impairment among children world-wide and a major public health problem in many indigenous populations. Early onset of hearing impairment compromise communication skills and social and psychological development, and monitoring the prevalence in a population is important to assess the need for interventions both at the school-, societal and clinical settings in a population.

Methods: 185 children from $5^{\text {th }}$ to $10^{\text {th }}$ grade from Sisimiut town and the nearby settlements participated in a clinical examination to have ear-examination and pure tone audiometry. Participants filled out a questionnaire at home with their parents before the clinical examination, and hearing impairment was collected as individual self-reports and as audiometric measurements. Ear examinations was assessed by two clinicians and if any discrepancy between self-reports and clinical measures they were reassessed.

Results: A total of 185 children between 9 and 15 years of age (median: 11 years, IQR: 10-13) were included, $60 \%$ ( $n=111)$ were girls. $247(70 \%)$ of the 355 available otoscopies were clinically assessed as normal. Cohen's Kappa coefficient was 0.31 . Eighteen children (10\%) were found to have hearing impairment using a PTA of $\geq 25 \mathrm{~dB}$ for either low or high frequencies or both as threshold in one or both ears. 32 children $(17 \%)$ reported hearing impairment to the extent that they were not able to keep up in school, of which half reported that it had lasted for more than one year. 7 of the 32 children reporting hearing impairment $(22 \%)$ reported that the extent of their hearing impairment was affecting their classroom experience so they were not able to follow. None of the children had hearing aids.

Conclusion: Self-reported and clinically screening for hearing impairment are two different concepts. Even though the two concepts are statistically correlated, the correlation coefficients are low. The contextual processing in which perceived hearing impairment is assessed in a social environment seems to conceptually differ from the measure of hearing by pure tone audiometry. Since both measure hearing impairment, the choice of measure should relate to the purpose and method of the investigation.

\section{Background}

Ear-infections is the leading cause of hearing impairment among children worldwide and a major public health problem in many indigenous populations $(1 ; 2)$. Studies have shown that early onset of hearing impairment compromise communication skills, academic performance, psychosocial behavior and emotional development $(3 ; 4)$, but studies are based on clinical examinations and not from representative studies of populations as studies on self-reported hearing impairment are scarce.

Greenland is known as a high risk population with regards to middle-ear infections (1), and concomitant conductive hearing loss. Mid-nineties studies of the young Greenlandic population found that $43 \%$ of children age 5 to 14 had hearing thresholds exceeding hearing loss (5). More recent data has been collected in a study from 2010 where 223 Greenlandic children age 4 to 10 years old was examined, and 
found that $20 \%$ had middle-ear infections at some point in childhood whereof $91 \%$ had developed some degree of permanent hearing loss $(6 ; 7)$. Jensen and colleagues studied 438 individuals from age 11 to 24 , and found hearing loss between $2 \%$ and $50 \%$ depending on the use of either the American SpeechLanguage-Hearing Association (ASHA) or World Health Organization (WHO) definition at that time (2011) (7). According to the former WHO criteria a person had a hearing loss if the pure tone average (PTA) was worse than 25 decibel $(\mathrm{dB})$ in the better ear. The current WHO definition states that a person with a PTA worse than $20 \mathrm{~dB}$ in any ear has a hearing loss (8).

A recent study examining 185 children age 9 to 15 , and found that $29 \%$ had hearing impairment using PTA of $15 \mathrm{~dB}$ for either low or high frequencies or both as threshold, and $10 \%$ had hearing impairment when using $25 \mathrm{~dB}$ as threshold (9)

Questionnaire-based data have been collected from Health Behavior in School-aged Children (HBSC) Greenland, which is a national cross-sectional survey among all school-children in Greenland from class 6 to 10, collected self-reported data on hearing impairment, and from data on 2.273 children age to 10 to $18,14 \%$ responded 'yes' to the question 'Does poor hearing prevent you from keeping up in class?" (10).

The research area of hearing disabilities in the Arctic region has historically received a lot of attention from both ear-nose-and-throat (ENT) doctors and epidemiologists. Whereas the former traditionally has a main focus on the pathophysiology of ear infection, treatment modalities and complications such as hearing loss, at the individual level, the latter has a main interest in studying the public health impact, the social, psychological and emotional consequences of a life with hearing impairment, as seen from a societal perspective. Public health advocates and policy makers have an interest of results from both groups of experts to optimally design interventions to support the individual for the benefit of the society, and to prevent public health at a broader level from worsening.

Reports of $10-20 \%$ of children having hearing impairment means that many individuals are suffering from compromised communication skills and worsening of psychosocial and emotional development $(3 ; 4)$. At a societal level, reported consequences has been of poorer reading skills, behavioral problems and lower IQ can affect the healthy development of future generations (4;11-14).

There are several ways to classify hearing impairment. Pure tone audiometry is considered the gold standard in population-based studies and screenings, but clinical measures vary according to definitions of threshold levels. Furthermore, audiometric outcomes can be limited by ambient noise, examiner competence and the developmental stage and behavior of the child.

Inconsistencies are also identified when using self-reported measures from questionnaires, as they can be limited by the validity of the questions, the child's motivation and interest in the questionnaire and the developmental stage and behavior of the child. The lack of self-report measures to use for children and adolescents - who may never have experienced normal hearing, and would therefore not be able to identify a change in hearing levels (from normal to impaired) - formed the development of items for a questionnaire-based survey among Greenlandic school-children (10). In 2017 to 2018 items were 
developed to include in the national questionnaire-based survey on adolescent health, HBSC Greenland. This study described the process of developing instruments available for epidemiological surveys to assess impaired hearing in a national sample (10). The present paper describes a validation of the developed items, by collecting information from individuals on both clinically assessed and self-reported impaired hearing screening, to test the association between the items developed for the questionnairebased survey and clinical measures of hearing impairment.

\section{Methods}

This present cross-sectional study included school-aged children in Sisimiut in Greenland. All children from 5th to 10th grade from the two schools in Sisimiut and the nearby settlements were invited to participate, corresponding to 422 children in total. Data was collected in September 2020.

Participants filled out a questionnaire with their parents or guardians before ear-examination and audiometry, and parent or guardian provided informed consent before taking part in the clinical examinations.

\section{Questionnaire}

The questionnaire was mainly constructed of questions from HBSC Greenland, which is the Greenlandic contribution to the international HBSC survey, including recently developed questions about hearing impairment and additional questions on social factors relevant to hearing impairment and risk factors for the development of hearing related diseases. The development of questions measuring hearing impairment has been described elsewhere (10). The questionnaire was available in Danish and Greenlandic.

As described by Schnohr et al. (2019) the item measuring self-reported hearing impairment was formulated; "Does poor hearing prevent you from keeping up in class?" with the response categories; Yes and no. If the respondent replied positively, the questionnaire would guide the respondent to three additional questions, with the following supplementary text; "You answered YES to poor hearing preventing you from keeping up in class:" and asking respondents to reply to the following question on extent of hearing impairment; "How much does poor hearing prevent you from keeping up in class?" with the response categories; A little, Quite a lot, and I can't keep up at all., a question on the source of knowledge to the hearing impairment; "How do you know you have poor hearing? (multiple answers allowed)" with five response categories; I noticed myself, Someone in my family told me, One of my teachers told me, One of my friends told me and A doctor/nurse/health visitor told me., and lastly a question on the duration of impaired hearing;"For how long have you suffered from poor hearing? with five response categories; Less than 3 months, More than 3 months but less than a year, 1-5 years, 5-10 year, and More than 10 years.

\section{Clinical examinations}


All examinations were conducted in a room in the schools, selected to reduce background noise. All respondents were examined for 15-30 minutes including a conversation about the questionnaire and an audiological examination. The audiological examination was conducted by a medical student (JSJ) who prior to data collection had received simulator based training and clinical teaching at the Department of Otorhinolaryngology, Head and Neck Surgery and Audiology, Rigshospitalet, Denmark. All examinations and conversations with respondents were followed by a Greenlandic research assistant who supported all work during the examinations, including connecting with school-children as respondents and before, during and after the inclusion into the study and the examination.

For the audiometry an Interacoustics Callisto ${ }^{\text {'m }}$ audiometer was used with a TDH39 headset. Air conduction (AC) thresholds were obtained at six frequencies: 250, 500, 1000, 2000, 4000, and $6000 \mathrm{Hertz}$ $(\mathrm{Hz})$. Thresholds were determined manually according to the ASHA guidelines \{American SpeechLanguage-Hearing Association, 2011232 /id\}.

Hearing impairment was categorized into with hearing impairment and without hearing impairment. The definition of hearing impairment was based on the PTA for the low frequencies (i.e., 500, 1000, and 2000 $\mathrm{Hz}$ ) and for the high frequencies (i.e. 4000 and $6000 \mathrm{~Hz}$. A child was considered to have a hearing impairment if the PTA for either the low or high frequencies were $>25 \mathrm{~dB}$ in any ear.

\section{Statistical Analyses}

Analyses was performed to assess the statistical association between self-reported (SR) and clinically measured (CM) hearing impairment.

A simple $2 \times 2$ table initially described the association between the SR and $\mathrm{CM}$, and false positives as well as false negatives were examined. Secondly Cohen's Kappa coefficient was calculated to assess the agreement between the two categorical items. The underlying assumption of using the Kappa coefficient is that the two categorical items tested are measuring the same construct.

Finally, univariate logistic regression examined SR by CM to assess the statistical association.

\section{Results}

A total of 185 (44\%) of the invited children between 9 and 15 years of age (median: 11 years) participated whereof $111(60 \%)$ were girls. Thirty-two of the 185 respondents answered 'yes' to the question whether they were not able to follow class because of poor hearing, corresponding to $17 \%$. When studying Table 1 further, it is worth highlighting that out of the 18 with clinically measured impaired hearing, almost half of them did not report a problem, and had not themselves recognized having a problem following school. Out of the 167 with normal hearing according to the clinical measures, $13 \%$ reported not to be able to follow school due to hearing problems. If reading the Table vertically, 22 out of the 32 students reporting impaired hearing as a cause of not being able to follow school was not classified as having a clinically noteworthy impaired hearing. Additionally, out of the 153 , who themselves reported normal hearing, $5 \%$ had impaired hearing from the audiological examinations (Table 1). 
Cohen's Kappa coefficient was 0.31 which indicates a very low agreement.

Univariate logistic regression showed an odds ratio (OR) of $8.2(95 \% \mathrm{Cl} 2.9-23.1)$ of SR when having been classified with hearing impairment by $\mathrm{CM}$.

\begin{tabular}{|lllll|}
\hline \multirow{2}{*}{$\begin{array}{l}\text { Table 1: Self-reported hearing impairment by clinically measured } \\
\text { hearing impairment }\end{array}$} & \multicolumn{3}{c|}{ Clinically measured } \\
\cline { 3 - 5 } & & Normal & Impaired & TOTAL \\
\hline Self-reported & Normal & 145 & 8 & 153 \\
\cline { 2 - 5 } & Impaired & 22 & 10 & 32 \\
\hline Simple Kappa Coefficient & TOTAL & 167 & 18 & 185 \\
\hline & & & $95 \%$ LCL & $\begin{array}{l}95 \% \\
\text { UCL }\end{array}$ \\
\hline & Kappa & & & 0,4979 \\
\hline
\end{tabular}

\section{Discussion}

If basing conclusions on impaired hearing among school-children in Sisimiut, the prevalence varies from $17 \%$ if based on self-reports, to $10 \%$ if based on clinical examination from the threshold chosen in the present survey. Choice of threshold levels determines the variation between the two types of measurement, and there are large inconsistencies identified when trying to associate the two measures.

A main finding of the present analyses is, that the traditional measures of correlation are best suited to illustrate that the two measures are not closely related. Kappa coefficient is used to measure inter-rater reliability, and taking account the possibility of an agreement occurring by chance. However, the underlying assumption of using Kappa is that the two measures are measuring the same, an assumption that must be rejected to hold.

With this finding, it becomes evident, that the clinical measures cannot be considered as an exact measure, and that researchers and clinicians should allow for a "grey zone" of classifications including the personal assessment of hearing impairment. This "grey zone" can be quantified by further analyses of the 30 out of 185 children that showed inconsistencies between the SR and CM, that were first evaluated for a second opinion, and initial findings were confirmed. Given the degree of variations, the best conclusion is to consider the two measurements of hearing impairment as covering substantially different areas, and the explanation could be found in that the one is a consequence of the other. This is supported by a strong statistical association of SR by CM, with an OR of 8.2.

As described by Schnohr et al. (2019) the item measuring self-reported hearing impairment was associated to the school-situation, since the item was developed for school-aged children. In the event, that a respondent was asked why he/she did not keep up in school, and the suggestion was made that is 
was due to impaired hearing, such a question can simply nudge the respondent to reply 'yes' even though the underlying cause - to not paying attention to school - is something else. Often children are asked to pay attention for other reasons than impaired hearing, and it would be relevant to adjust for those related causes. Age, gender, socioeconomic background and overall self-perceived health has been demonstrated to be associated with disagreement between self-reported hearing and audiometric outcome among older adults and may also affect the responses of children $(15 ; 16)$. However, in a sample size of 185 respondents, it is not statistically sound to examine associations of related predictors on impaired hearing, to e.g. study the school environment and other aspects of the respondent's health, but it would be interesting and relevant for future studies.

Important factors that could lead to self-reported hearing difficulties in school but a normal pure-tone audiogram at examination are auditory neuropathy spectrum disorders, auditory processing disorders and any attention deficit disorder. Furthermore, the acoustic environment in the school is important. Background noise in the classroom, size of the room, number and position of students will affect their ability to hear the teachers voice

In Greenland it is only possible - as in this study - to use pure tone audiometry. Hearing is a highly complex neurological process that involve not only the outer and inner ear but also lower and higher cerebral functions that are interacting and are influenced by psychological processes and by the broader context in which the hearing takes place. The individual perception of what is heard therefore is a meta process.

Considerations can be taken to what the measurement properties are for each of the two methods, and taking into account if the conclusions derived should stem from a representative result for the entire population - in which case an epidemiological survey of a large representative samples of the population is most appropriate - or whether researchers are looking for clinical conditions causing hearing impairment - in which case clinical examinations of high risk populations is most appropriate.

Among older populations audiometric measures was found to explain less than $50 \%$ of the variance in hearing handicap and it was suggested that hearing handicap in the elderly will be measured more appropriate via a self-report format rather than as an inference from audiometric data (17)

In spite of clear findings that the two measurements are not measuring the same construct, it is clear that they are closely related. Irrespectively where the threshold levels are set, it is fair to presume that students that are classified as normal hearing will have problems following school, and it becomes evident that both measures have each their relevance, depending on the purpose and method of any given investigation. Based on the present study, it is not appropriate to conclude whether the self-reported or the measured collected from clinical examinations have the highest reliability, as both measurements have uncertainties, and there is no golden standard available at present.

In any case, the Arctic population is a high risk population for chronic conditions related to impaired hearing, and a continued focus on the monitoring and examinations of both representative and high-risk 
populations serves as an important topic for future public health in Greenland.

\section{Conclusions}

The two measurements of hearing impairment cover substantially different areas, and the explanation could be found in that the one is a consequence of the other as there are large inconsistencies identified when correlating the two measures. Since both measure hearing impairment, the choice of measure should relate to the purpose and method of the investigation.

Future studies will examine the causes of self-reported impaired hearing and include clinically measured hearing impairment as one of the explanatory factors.

\section{Abbreviations}

ASHA

American Speech-language Hearing Association

WHO

World Health Organization

PTA

Pure Tone Audiometry

$\mathrm{dB}$

decibel

HBSC

Health Behavior in School-aged Children

ENT

ear-nose-throat

AC

Air Conduction

SR

self-reported

$\mathrm{CM}$

clinically measured

OR

odds ratio

$\mathrm{LCL}$

lower confidence limit

UCL

upper confidence limit

\section{Declarations}




\section{Ethics approval and consent to participate}

This study followed the Helsinki declaration and was approved by ethical committee in Greenland (KVUG 2020-10). All participants gave informed consent.

\section{Consent for publication}

Not applicable

\section{Availability of data and materials}

The dataset used and analysed during the current study are available from the corresponding author on request

\section{Competing interests}

All authors declare that they have no competing interests

\section{Funding}

This study received financial support from William Demant Fonden (Grant number: 20-1001), Dagmar Marshalls Fond (Grant number: 500020), and Mauritzen la Fontaine Fonden (no grant number applicable). The funds had no role in the study, design or interpretation of data.

\section{Author's contributions}

CS and RGJ designed the study and JSJ collected data. JBJ and RGJ interpreted all clinical examinations twice, CS performed all analyses and was a major contributor in writing the manuscript. All authors contributed with substantial comments and suggestions, and all authors read and approved the final manuscript.

\section{Acknowledgements}

The authors are thankful to all participating children and their parents in Sisimiut and the settlements Itelleq and Sarfannguit, and the staff at the schools for their cooperation and advice. The authors also wish to thank the technicians at the department of Audiology, Rigshospitalet, for help in preparing equipment for the examinations, and the staff at the Sisimiut Health Center for support in the field work.

\section{References}

1. Bluestone CD. Epidemiology and pathogenesis of chronic suppurative otitis media: implications for prevention and treatment. Presented at the World Health Organization \& CIBA Foundation Joint Workshop on Prevention of Hearing Impairment from Cronic Otitis Media, Foundation CIBA, 
London,November 1996.1. International Journal of Pediatric Otorhinolaryngology 1998;42(3):207 23.

2. Jensen RG, Homøe P, Andersson MF, Koch A. Long-term follow-up of chronic suppurative otitis media in a high-risk children cohort. Int J Pediatr Otorhinolaryngol. 2011;75(Electronic):1872-8464. 948 54.

3. Bess FH, Dodd-Murphy J, Parker RA. Children with minimal sensorineural hearing loss: prevalence, educational performance, and functional status. Ear Hear. 1998;19(5):339-54.

4. Hall AJ, Maw R, Midgley E, Golding J, Steer C. Glue ear, hearing loss and IQ: an association moderated by the child's home environment. PLOS One. 2014;9(2):e87021.

5. Homøe P, Christensen RBFAU, Breatlau P. Hearing in elementary school children in Nuuk and Sisimiut, Greenland. Arctic Med Res. 1995;54(3):145-50.

6. Avnstorp MB, Homøe P, Bjerregaard P, Jensen RG. Chronic suppurative otitis media, middle ear pathology and corresponding hearing loss in a cohort of Greenlandic children. Int J Pediatr Otorhinolaryngol. 2016;83:148-53.

7. Jensen $R G$, Koch $A F$, Homøe P. The risk of hearing loss in a population with a high prevalence of chronic suppurative otitis media. Int J Pediatr Otorhinolaryngol. 2013;77(9):1530-5.

8. World Health Organization. Deafness and hearing loss 2021. https://www.who.int/healthtopics/hearing-loss\#tab=tab_1. Accessed March 25th 2021.

9. Jensen JS, Schnohr C, Skovsen CF, Homøe P, Jensen RG. Prevalence of hearing loss among schoolaged children in Greenland. Submitted to International Journal of Otorhinolaryngology. In press 2021.

10. Schnohr C, Frid KC, Jensen RG, Homøe P, Niclasen BVL. Self-reported hearing impairment among Greenlandic adolescents: Item development and findings from the Health Behaviour in School-aged Children study 2018. Int Jr Pediatr Otorhinolaryngol. 2019 Nov;126:109629.

11. Fougner V, Kørvel-Hanquist A, Koch A, Dammeyer J, Niclasen J, Lous J, et al. Early childhood otitis media and later school performance - A prospective cohort study of associations. Int J Pediatr Otorhinolaryngol. 2017;94:87-94.

12. Golz A, Westerman ST, Westerman LM, Gilbert DA, Netzer A. Does otitis media in early childhood affect reading performance in later school years? Otolaryngol Head Neck Surg. 2006;134(6):936-9.

13. Hall WC, Li D, Dye TDV. Influence of Hearing Loss on Child Behavioral and Home Experiences. Am J Public Health. 2018;108(8):1079-81.

14. Niclasen J, Obel C, Homøe P, Kørvel-Hanquist A, Dammeyer J. Associations between otitis media and child behavioural and learning difficulties: Results from a Danish cohort. Int J Pediatr Otorhinolaryngol. 2016;84:12-20.

15. Curti SA, Taylor EN, Su D, Spankovich C. Prevalence of and Characteristics Associated With Selfreported Good Hearing in a Population With Elevated Audiometric Thresholds. JAMA Otolaryngol Head Neck Surg 2019 Jul 1;145(7):626 - 33. 
16. Tsimpida D, Kontopantelis E, Ashcroft D, Panagioti M. Comparison of Self-reported Measures of Hearing With an Objective Audiometric Measure in Adults in the English Longitudinal Study of Ageing. JAMA Netw Open 2020 Aug 3;3(8):e2015009.

17. Weinstein BE, Ventry IM. Audiometric correlates of the Hearing Handicap Inventory for the elderly. $J$ Speech Hear Disord 1983 Nov;48(4):379-84.

\section{Supplementary Files}

This is a list of supplementary files associated with this preprint. Click to download.

- STROBEchecklistcrosssectional.docx 